\title{
Epicardial fat mimicking left atrial appendage thrombus
}

\author{
Nicolas Johner ${ }^{1}$, Philippe Maziarski ${ }^{1}$, Jean-Paul Vallée ${ }^{2}$, Philippe Meyer $^{1}$ \\ ${ }^{1}$ Cardiology Service, Geneva University Hospitals, Geneva, Switzerland \\ ${ }^{2}$ Radiology Service, Geneva University Hospitals, Geneva, Switzerland
}

A 77-year-old woman was hospitalized for acute decompensated heart failure due to paroxysmal atrial fibrillation (AF) with rapid ventricular response. Transesophageal echocardiography (TEE) was performed prior to electrical cardioversion because a left atrial appendage (LAA) thrombus had been described 3 years earlier. Right next to the left atrium, an echogenic, multilobulated, partially mobile mass, similar to the one previously reported, was visualized (Fig. 1A, B; Suppl. Video 1). However, on closer examination, the cavity surrounding the mass was not continuous with the left atrium. By slightly rotating the TEE probe the true LAA lumen was found, thrombusfree, but collapsed by an echogenic mass (Fig. 1C; Suppl. Video 2). Computed tomography (CT) showed a thin tubular thrombus-free LAA lumen surrounded by isodense fluid in the left pulmonary artery recess of the transverse pericardial sinus and by hypodense epicardial fat (Fig. 1D).

The pericardial cavity consists of the pericardial cavity proper, transverse sinus and oblique sinus. The LAA is adjacent to two pericardial recesses: the left pulmonary artery recess of the transverse sinus, and the left pulmonary vein recess of the pericardial cavity proper. These recesses exist in $60-80 \%$ of the population and exhibit substantial inter-individual variability. The LAA itself has a variable shape and size, with $80 \%$ of the population presenting $\geq 2$ lobes. Therefore, TEE examination of the LAA should include multiplanes to distinguish pericardial recesses from the LAA lumen. In the present case, epicardial fat surrounding a large fluid-filled left pulmonary artery recess was initially mistaken for an LAA thrombus, precluding electrical cardioversion.

Conflict of interest: None declared

Address for correspondence: Philippe Meyer, MD, Cardiology Service, Geneva University Hospitals, Rue Gabrielle-Perret-Gentil 4, 1205 Geneva, Switzerland, tel: +41-22 372 95 97, fax: +41-22 37237 45, e-mail: philippe.meyer@hcuge.ch 


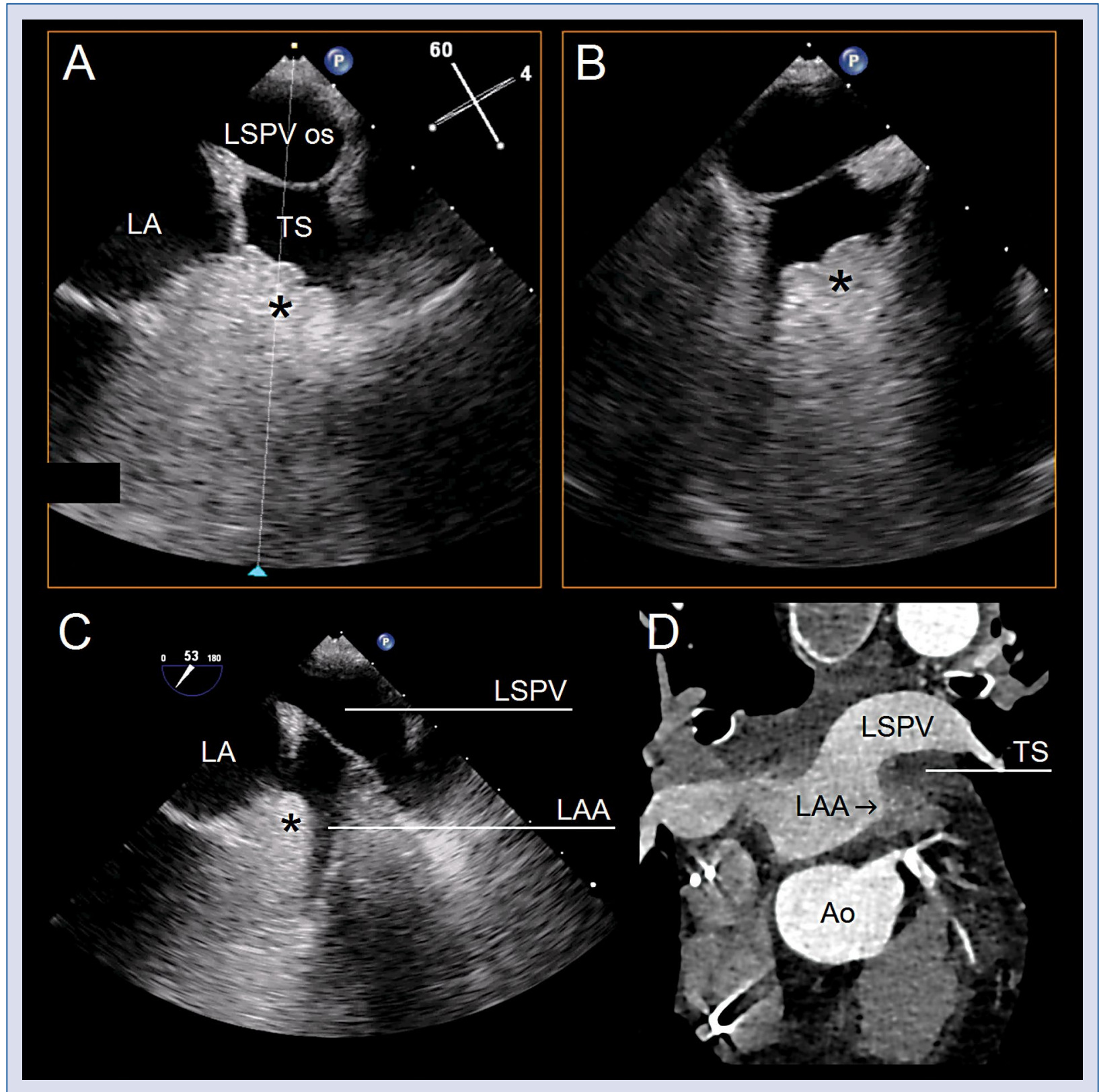

Figure 1. A, B. Biplane transesophageal echocardiography, mid-esophageal view. An echogenic multilobulated mass (asterisk), in the fluid-filled left pulmonary artery recess of the pericardial transverse sinus mimics a thrombus in the left atrial appendage (LAA); C. Transesophageal echocardiography, mid-esophageal view. The true LAA lumen is in continuity with the left atrium (LA) and appears collapsed by surrounding echogenic tissue (asterisk); D. Reconstruction of a transversal contrast-enhanced cardiac computed tomography in the same orientation as in panel C. The collapsed LAA lumen (arrow) does not contain any intraluminal mass. Posteriorly, the left pulmonary artery recess of the transverse pericardial sinus is filled with an isodense material compatible with fluid surrounded by hypodense epicardial fat; Ao - aorta; LSPV - left superior pulmonary vein; TS - left pulmonary artery recess of the transverse pericardial sinus. 